\title{
A comparative analysis of several material models in LS-DYNA at high velocity impact
}

\author{
Arina Bakulina ${ }^{1}$ and Andrey Buzyurkin ${ }^{1, *}$ \\ ${ }^{1}$ Khristianovich Institute of Theoretical and Applied Mechanics of Siberian Branch of RAS, \\ 4/1 Instituskaya str., Novosibirsk, 630090, Russia
}

\begin{abstract}
The questions of application of the LS-DYNA finite element analysis package for describing the interaction of a spherical projectile and a massive metal obstacle are considered. Based on a comparison of the results of numerical simulation with experimental data, a qualitative possibility has been shown to determine the magnitude of the dynamic hardness of the obstacle material.A simulation of the collision of a spherical impactor and a massive metal barrier shaped as a cube is performed. Data are obtained on the penetration of the striker into the barrier.Using the simulation curve, the values of the dynamic hardness of the obstacle were restored and a comparison was made with their experimental values. The qualitative correspondence is shown.
\end{abstract}

\section{Introduction}

To describe the complex phenomena that occur during the collision of deformable bodies with a high relative speed, it is necessary to know the mechanical characteristics of materials in a wide range of loading speeds. Accurate material properties are required for calibrating finite element models to ensure the reliability of numerical simulation results. Numerical simulation using the finite element method (FEM) is a fairly effective way to test complex structures and is used in many modern design systems. Despite the fact that most loading processes are associated with the elastic reaction of a mechanical system, there are many applications that require modeling of the system taking into account plastic deformation. Until the yield point is reached, the linear dependence of stress on strain is well known, easily determined, and has a widely available base of material properties. Compared to plastic deformation, the linear dependence is less dependent on the strain rate, temperature, and anisotropy of the material. Until the yield point is reached, the molecular bonds of the material are sex by tensile stresses. In this case, irreversible damage to the material does not occur. Upon reaching the yield strength, the material no longer deforms linearly, since molecular bonds begin to break, resulting in the appearance of irreversible damage. Plastic deformation is a complex process, depending on temperature, strain rate, anisotropy of material properties, residual stresses and many other factors.

Application packages, such as LS-DYNA or ABAQUS, can be used to model the behavior of materials under dynamic loading beyond the yield strength. These applications

\footnotetext{
*Corresponding author: buzjura@itam.nsc.ru
} 
require complex material behavior models that depend on the stress state, strain rate, and temperature. One of the most important constituent parts of these packages, which determine the reliability of the calculations, are the defining relations used in them (mathematical models of behavior) and the criteria for destruction. Since these models are used to calculate the behavior of the material beyond the yield strength, plasticity studies play an important role in the development of both the package itself and the properties of the materials studied. To equip behavior models and fracture criteria with the necessary parameters and constants, an extensive base on the dynamic properties of materials is required. In addition, to verify the adequacy of the models used, it is necessary to develop and conduct some "model" field experiments.

\section{Numerical Simulation}

To select the preferred material models, which were implemented in the LS-DYNA software package, which describe the destruction of the impactor and barriers during highspeed impact, a number of models were selected to describe the processes of deformation, flow, hardening and fracture of metal materials from the entire list of available materials in LS- DYNA. The main selection criteria were:

- applicability of the material model for metals and alloys;

- the presence in the model of the ability to describe materials at strain rates up to $10^{5}$ $1 / \mathrm{s}$;

- the presence in the model of fracture criteria or the possibility of an additional description of the behavior of the material during fracture;

- description model capabilities hardening and spalling, i.e. processes and phenomena occurring in metals at high strain rates.

Of the more than 200 material behavior models implemented in the LS-DYNA software package, the MAT_PLASTIC_KINEMATIC (MAT_003) model was selected as the test model. For the subsequent simulation of the collision process, we selected material models such as

*MAT_POWER_LAW_PLASTICITY (MAT_018),

*MAT_PIECEWISE_LINNEAR_PLASTICITY $($ MAT_024)

*MAT_SIMPLIFIED_JOHNSON_COOK (MAT_098).

These material models fully satisfy the specified criteria.

In addition to the fact that we had to choose from a variety of options the models of material behavior that were suitable for us according to the criteria, we also needed to choose the metal or alloy suitable for the computational experiment. To conduct a test experiment, we selected mild steel. After the successful completion of the test experiment with the low-carbon steel grade A570 Grade 36 [1], we took the carbon Steel 20 (AISI1020) for subsequent calculations.

To describe the contact interaction between the projectile and the obstacle, the *CONTACT_ERODING_SURFACE_TO_SURFACE map was chosen, in which the criterion for destruction in the contact gives an additional advantage, removing the damaged elements from the calculation.

In a number of experiments [2], carried out in a wide range of impact velocities of a projectile and an obstacle for different types of metals, a relationship was established between the penetration depth $L_{k}$ related to the initial diameter of the spherical projectile $d_{0}$ and the dimensionless parameter $\frac{\rho_{0} V_{0}^{2}}{H_{1}}$, where $\rho_{0}$ is the density of the projectile 
material and the obstacle, $V_{0}$ is the initial velocity of the projectile, $H_{1}$ is the dynamic hardness determined experimentally $[3,4]$.

In order to determine the parameter $H_{1}$ numerical simulation of impact of the obstacle at high-speed collision with the steel impactor was carried out using finite element package LS-DYNA [5]. LS-DYNA has a wide range of options for describing the behavior of elastoplastic materials under dynamic loads, but the choice of constants for a particular material is not an easy task, as it might seem at first glance [6-8].The problem was solved for $3 \mathrm{D}$ case in the Lagrangian formulation. Geometric model (see Fig. 1) was corresponded to the experimental. The spherical projectile and the obstacle, made of steel and having a density of $7850 \mathrm{~kg} / \mathrm{m}^{3}$, are considered. The material properties in the course of the work were set according to the experimental data [2]. In the initial data, the diameter of the spherical projectile is $10 \mathrm{~mm}$. The obstacle is a massive cube, the dimension of which exceed the dimensions of the impactor by a factor of 10 and amount to $100 \times 100 \times 100 \mathrm{~mm}$. To analyze the behavior of a steel projectile in a collision with a steel obstacle, the velocity range of impact was taken from 250 to $1000 \mathrm{~m} / \mathrm{s}$.

In [9] have calculated the low strain-rate material property coefficients with plastic failure strain for all 1,044 materials in the Database so that they can be used in LS-DYNA.

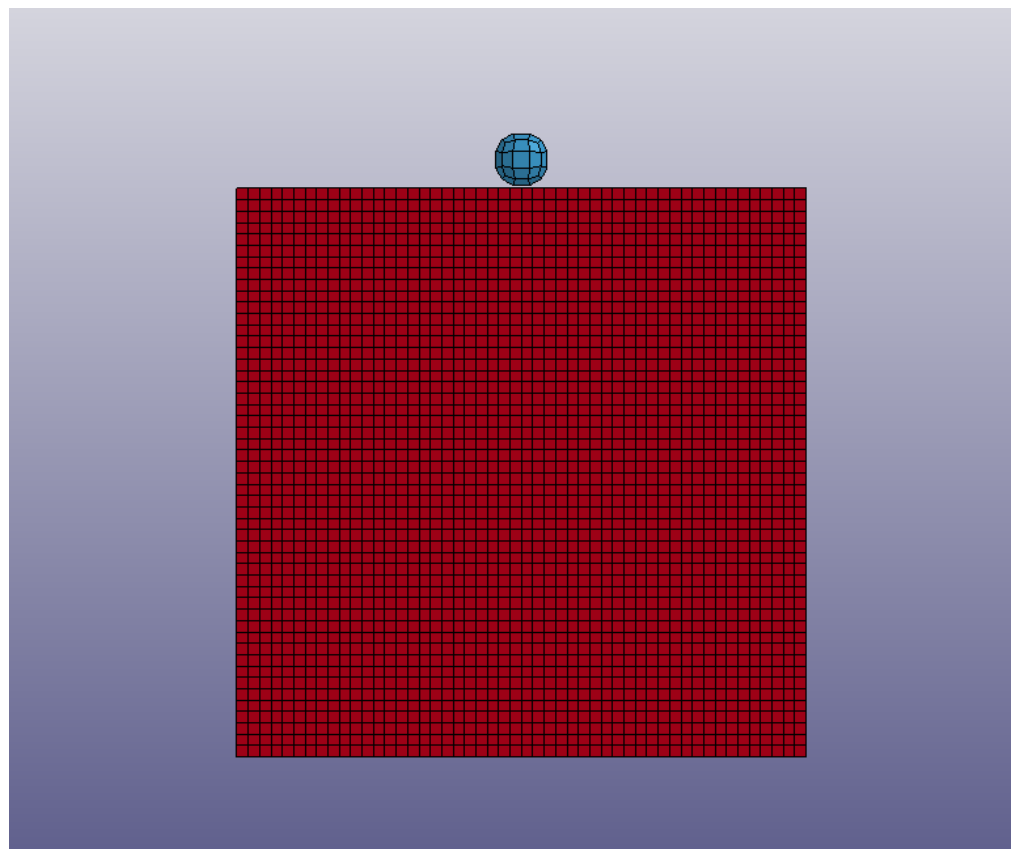

Fig. 1. The initial position of the spherical impactor and the massive metal barrier

Mesh size convergence tests have been performed to find out the acceptable mesh size. In such analysis the element size in each body was decreased (from 50 to 200 cells per edge) and the problem was solved again and if the results are nearly similar therefore it can say the first mesh is good enough and results do not depend on the mesh size.

The results of calculations of the introduction of a steel impactor into a massive barrier using various types of mesh and material models are presented in (Figs. 2 and 3). 


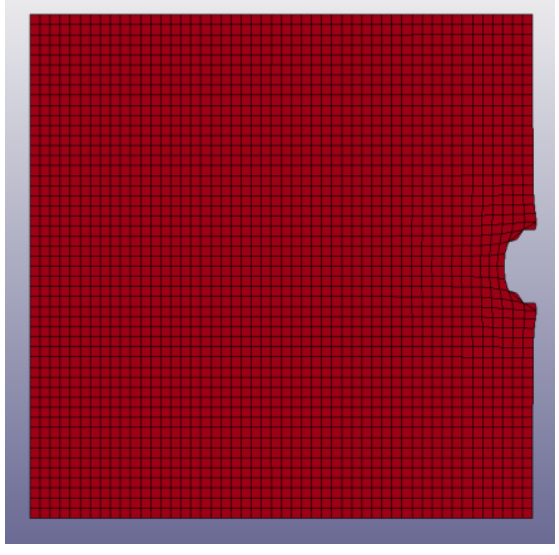

MAT_003

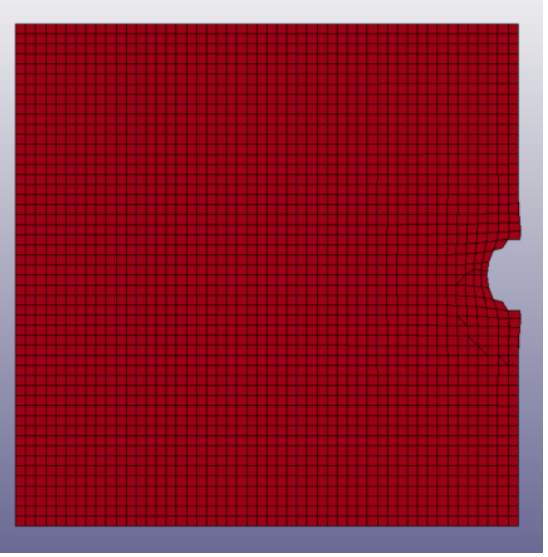

MAT_024

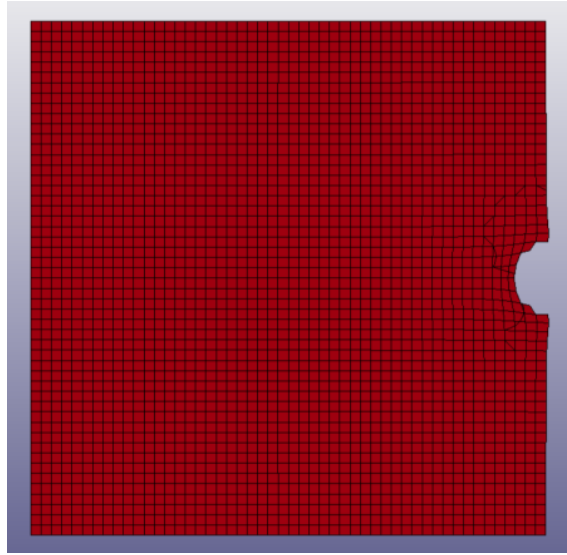

MAT_018

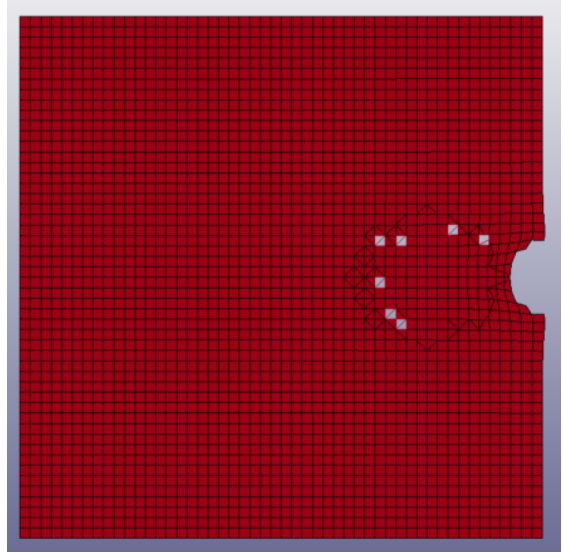

MAT 098

Fig. 2. Depth of penetration. $V_{0}=1000 \mathrm{~m} / \mathrm{s}$. Mesh size $=50$ 


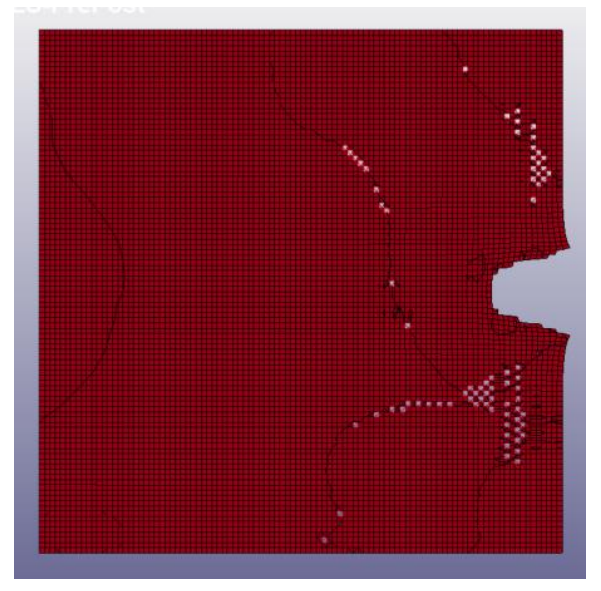

MAT_003

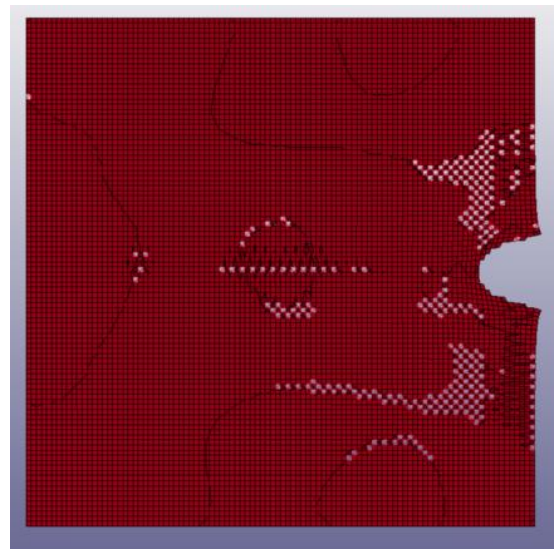

MAT_024

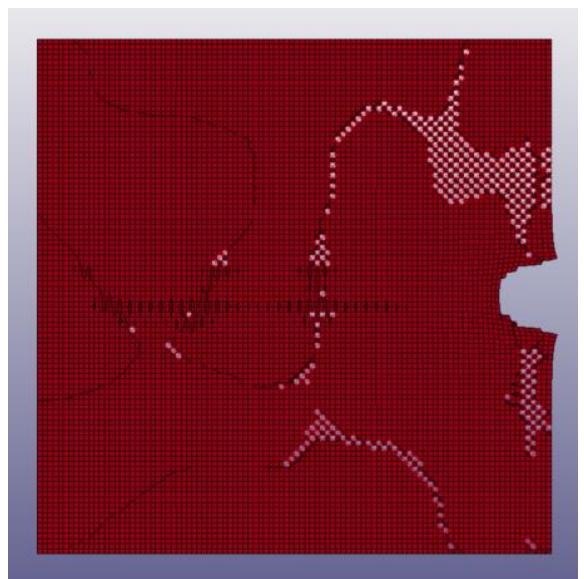

MAT_018

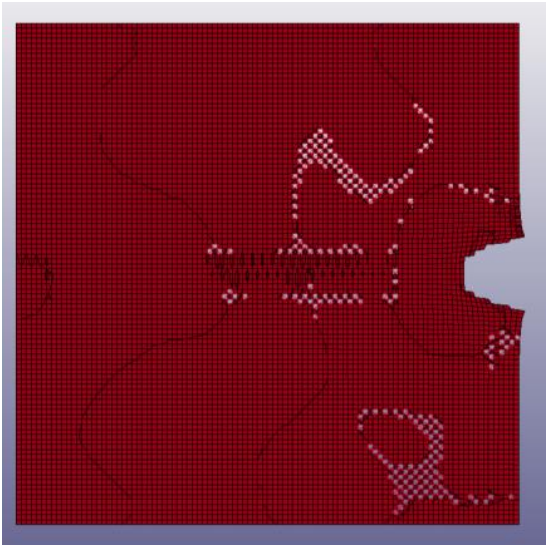

MAT_098

Fig. 3. Depth of penetration. $V_{0}=1000 \mathrm{~m} / \mathrm{s}$. Mesh size $=100$

Table 1 shows data on the ratio of penetration depth to the initial diameter of the impactor depending on the type of mesh and material model, as well as the dimensionless parameter $\frac{\rho_{0} V_{0}^{2}}{H_{1}}[2]$. 
Table 1. The ratio of penetration depth to the initial diameter of the impactor depending on the type of mesh and material model.

\begin{tabular}{|c|c|c|c|c|}
\hline $\begin{array}{c}\text { Material } \\
\text { model }\end{array}$ & Mesh & $L_{k}$ & $L_{k} / d_{0}$ & $\frac{\rho_{0} V_{0}^{2}}{H_{1}}$ \\
\hline \multirow{3}{*}{ MAT_003 } & 50 & 0.00573 & 0.573 & 5.6 \\
\cline { 2 - 5 } & 100 & 0.01356 & 1.356 & 28 \\
\hline \multirow{3}{*}{ MAT_018 } & 200 & 0.01152 & 1.152 & 19 \\
\cline { 2 - 5 } & 100 & 0.00620 & 0.62 & 5.99 \\
\cline { 2 - 5 } & 200 & 0.01011 & 1.011 & 14.5 \\
\hline \multirow{3}{*}{ MAT_024 } & 50 & 0.0112 & 1.12 & 18 \\
\cline { 2 - 5 } & 100 & 0.01105 & 0.618 & 17.6 \\
\hline \multirow{3}{*}{ MAT_098 } & 200 & 0.0112 & 1.105 & 18 \\
\cline { 2 - 5 } & 100 & 0.01100 & 1.12 & 5.9 \\
\cline { 2 - 5 } & 200 & 0.0112 & 1.1 & 18 \\
\hline
\end{tabular}

The calculated values of dynamic hardness $H_{1}$, calculated on the basis of the obtained values of the dimensionless parameter $\frac{\rho_{0} V_{0}^{2}}{H_{1}}$, for various values of $L_{k} / d_{0}$ are given in Table 2. It can be seen that with decreasing mesh size at a speed of $1000 \mathrm{~m} / \mathrm{s}$, the value of dynamic hardness is almost the same

Table 2. The calculated values of dynamic hardness $H_{1}$, for various speeds, mesh size and type of material (the beginning).

\begin{tabular}{|c|c|c|c|c|}
\hline \multirow{3}{*}{$V_{0} \cdot \mathrm{m} / \mathrm{s}$} & \multirow{3}{*}{ Material model } & \multicolumn{3}{|c|}{$H_{1}, \mathrm{MPa}$} \\
\hline & & \multicolumn{3}{|c|}{ Mesh } \\
\hline & & 50 & 100 & 200 \\
\hline \multirow{4}{*}{250} & MAT_003 & 1165.77 & 960.05 & - \\
\hline & MAT_018 & 1165.77 & 532.2 & - \\
\hline & MAT_024 & 1165.77 & 532.2 & - \\
\hline & MAT_098 & 1165.77 & 532.2 & - \\
\hline \multirow{4}{*}{500} & MAT 003 & 1025.39 & 365.39 & - \\
\hline & MAT_018 & 1332.31 & 476.52 & - \\
\hline & MAT_024 & 1440.07 & 476.52 & - \\
\hline & MAT 098 & 1332.31 & 476.52 & - \\
\hline
\end{tabular}


Table 2. The calculated values of dynamic hardness $H_{1}$, for various speeds, mesh size and type of material (the ending).

\begin{tabular}{|c|c|c|c|c|}
\hline \multirow{3}{*}{$V_{0} \cdot \mathrm{m} / \mathrm{s}$} & \multirow{3}{*}{ Material model } & \multicolumn{3}{|c|}{$H_{1}, \mathrm{MPa}$} \\
\hline & & \multicolumn{3}{|c|}{ Mesh } \\
\hline & & 50 & 100 & 200 \\
\hline \multirow{4}{*}{750} & MAT_003 & 728.37 & 284.85 & - \\
\hline & MAT_018 & 1006.08 & 480.02 & - \\
\hline & MAT_024 & 972.76 & 480.02 & - \\
\hline & MAT_098 & 972.76 & 470.29 & - \\
\hline \multirow{4}{*}{1000} & MAT_003 & 1160.59 & 241.49 & 410.5 \\
\hline & MAT_018 & 1052.96 & 457.59 & 433.3 \\
\hline & MAT_024 & 1052.96 & 386.86 & 433.3 \\
\hline & MAT_098 & 1052.96 & 393.87 & 433.3 \\
\hline
\end{tabular}

\section{Conclusion}

Based on the calculation results, the following remarks can be made

- dynamic hardness is a function of the strain rate;

- it is necessary to conduct several experiments for different materials in order to gain a better understanding of the behavior of dynamic hardness over a wide range of strain rates.

This work was carried out within the framework of the Program of Fundamental Research of the State Academies of Sciences in 2013-2020 (Project No. AAAA-A17-117030610136-3).

\section{References}

1. A.E. Buzyurkin, "Modeling the interaction of a deformable projectile with a metal obstacle using LS-DYNA package," AIP Conf. Proc. 2027, 030163 (2018)

2. N.A. Zlatin, A.P. Krasilschikov, G.I. Mishin, and N.N. Popov, Ballisticheskiye Ustanovki i Ikh Primeneniye v Eksperimental'nykh Issledovaniyakh [Ballistic Installations and Their Application in Experimental Studies] (Nauka, 1974) (in Russian)

3. F.F. Vitman and N A. Zlatin, Sov. Phys. Tech. Phys. 33, 8, 982 - 989 (1963)

4. L.V. Belyakov, F. F. Vitman, and N.A. Zlatin, Sov. Phys. Tech. Phys. 33, 8, 990 - 95 (1963)

5. J. Hallquist, $L S-D Y N A \circledR$ Theory Manual (March ,2006)

6. Y. Xia, C. Zhang, L. Zhang, W. Shen, and Q. Xu, J. Mater. Res. 32, 20, 3875 - 3884 (2017)

7. A.E. Buzyurkin, I.L. Gladky, and E.I. Kraus, J. Appl. Mech. Tech. Phys. 56, 2, 330 $336(2015)$

8. Y. Li, H. Ji, Z. Cai, X. Tang, Y. Li, and J. Liu, Materials (Basel). 12, 12, 1893 (2019).

9. "Varmintal," https://www.varmintal.com/aengr.htm\%0A. 